\title{
Analysis of Hybrid Technique for Motion Planning of Humanoid NAO
}

\author{
Abhishek Kumar Kashyap a,1, , Dayal R. Parhi a,2, Anish Pandey b,3 \\ ${ }^{a}$ Robotics Laboratory, Mechanical Engineering Department, National Institute of Technology, Rourkela 769008, Odisha, \\ India \\ ${ }^{\mathrm{b}}$ School of Mechanical Engineering, Kalinga Institute of Industrial Technology, Bhubaneswar-751024, Odisha, India \\ ${ }^{1}$ techmech46@gmail.com; ${ }^{2}$ dayalparhi@gmail.com; ${ }^{3}$ anish.pandeyfme@ kiit.ac.in \\ * Corresponding Author
}

ARTICLE INFO

Article history

Received 15 March 2021

Revised 23 March 2021

Accepted 26 March 2021

Keywords

Humanoid NAO;

Webots;

Eagle strategy;

Ant colony optimization

\begin{abstract}
The navigation of a humanoid robot is essential because it is the basic requirement of any assigned task. Singly used motion planning techniques may take a long path to reach the target and increase the computational cost. Therefore, in this article, a hybrid controller is employed in the humanoid NAO for motion planning assignment. The Eagle strategy (ES) with Ant colony optimization (ACO) is introduced in this article for evaluating precise steering angles for humanoid robots as they traverse a route from a reference point to a target point. This enables the robot to achieve its specific target more quickly by avoiding barriers and obtaining the minimal global direction. The hybridized ESACO approach is critical in determining precise steering angles to escape obstacles. The details of terrain are obtained using vision and ultrasonic sensors, which also include the barriers ranges to the ES as an input variable. The ES's input parameters are the barrier ranges from the NAO in front, left, and right directions and the technique's output variable is the precise steering angle. The designed controller is tested in both a simulation and an experimental setting with a variety of obstacles. The outcomes of both simulation and experimental conditions are compared, and a strong correlation is identified in those with the fewest deviations.
\end{abstract}

This is an open-access article under the CC-BY-SA license.

\section{Introduction}

The humanoid NAO with 25 degrees of freedom is created by Aldebaran robotics [1] is used in the study. Humanoid robots are gaining importance in investigation these days because they resemble humans and can efficiently operate in factories while minimizing human work. The humanoid's gesture control approach must be successful. As a result, route planning for humanoids is a difficult research project for scientists. In this article, an improved Eagle strategy is employed for trajectory planning research using ACO that takes input data and outputs a variable to the robot for seamless navigation of a single humanoid in terrain with 
complex obstacles. So many scientists have utilized a variety of optimization strategies [2] for robot navigation, some of which are described below.

Many algorithms are implemented in mobile robots for path optimization and humanoid robot for gesture control [3][4][5][6]. Murray and Jennings [7] have proposed a visual guidance approach for the path planning of a mobile robot in a cluttered environment. The strategy encompasses the use of a stereo vision-based approach for defining the path of a robot by the generation of in-depth images of the obstacles and the target. The proposed method proves to be a suitable alternative to the commonly used infrared and laser sensors. Deepak et al. [8] have proposed the usage of an adaptive learning strategy during path planning of a mobile robot. The navigational strategy presented here is the culmination of adaptive control and the innate immune-based path planner to sketch out an effective path for the robot in unknown environments. Meyer and Filliat [9] have emphasized the application of path planning strategies in various discretized and continuous environments via the integration of paths between the initial and final points. Various adaptive strategies for unknown environments and their effectiveness are discussed in the paper. Kowdiki et al. [10] have emphasized the path planning of a series of mobile robots where the leader robot predicts the path via APF algorithm and the follower bots adapt to the pathway already set by controlling the turning angle and the separation distance. Zhao [11] has emphasized the modifications of the potential field functions in the given terrain. The attractive potential function varies according to the minimum separation between the robot and the obstacle, whereas the repulsive potential function considers the relative position between the robot and the final target. The approach proves to be sufficiently efficient in clearing the robot out of the local minima zone. Sfeir et al. [12] have focused on the implementation of a modified repulsive function in the sample space to reduce oscillations and maintain the course of the robot collision-free. Further, integration of the rotational forces on the robot helps in obtaining a smoother turning trajectory.

Choset and Pignon [13] have emphasized a specific cell decomposition strategy with the primal purpose of coverage of the environment provided to the humanoid robot. The main objective attained by this algorithm is the generation of an efficient and robust path for the humanoid to traverse successfully from the initial to the final position. Kavraki et al. [14] have demonstrated the motion planning of a mobile robot in a static environment using two phases of algorithm-learning phase and query phase. The learning phase sketches the basic node connections between various cells to draw an obstacle-free path for the robot, while the query phase guidelines it to connect the dots between the initial and final positions based on various parameters. Cargill [15] has presented a complete analysis and check on the robustness and efficiency of the dining philosophers problem via test cases. On complete examination, the method proves to be aptly suited for application in synchronization problems with minor weakness declared. Wedde [16] has demonstrated the solution of the dining philosophers problem via the usage of loosely coupled systems. The complete demonstration shows the factors affecting the behavior of the approach in a stepwise manner and the directions predicted by the approach to move out of a local state.

According to the findings of the literature review, humanoid robot gesture control is constrained. Various researchers have also implement hybrid controllers [3][17][18][19][20][21] for navigation of mobile and humanoid robots but are constraint in numbers. As a result, the present study attempts to create a suitable hybrid ES-ACO controller for humanoid robot route mapping research. The established controller has been evaluated with a single humanoid in both a simulation and an experimental situation, yielding acceptable findings 


\section{The Proposed Controller}

\subsection{Eagle Strategy}

The Eagle strategy (ES) has been influenced by an eagle's fodder activity, which flies randomly as the Levy flights [22]. The flowchart of the ES technique for the route mapping of the humanoid robot is described in Fig. 1. ES approach has been employed to manage local hunts for planning assignments using a number of optimization techniques. This is identical to the arbitrary reset approach of hill climbing, but two distinctions are present. The Eagle Strategy is a two-stage variability approach for global search and intense local search. The Levy walks used by the Eagle Strategy approach to further analyze the global quest arena. Eagle Strategy is built upon Levy flights for the global $n$-dimensional scan. An active local colony optimizer (ACO) is employed to identify the possible response for local searches. The ACO approach can examine locally. With new global quests in the various areas, the hunting mechanism is repeated. The ES approach included the advantages of the two techniques, PSO and $\mathrm{ABC}$, as seen as a case study in the experimental field in order to obtain better performance. The levy flights can be written as

$$
L_{d}(f): \frac{\lambda \Gamma(\lambda) \sin (\pi \lambda / 2)}{\pi} \frac{1}{f^{1+\lambda}},\left(f>>f_{0}>0\right),(1<\lambda \leq 3)
$$

where $\Gamma(\lambda)$ represents the standard gamma function and $f$ represents the step length. In order to make the Levy walks as Cauchy distribution, $\lambda=2$ is employed.

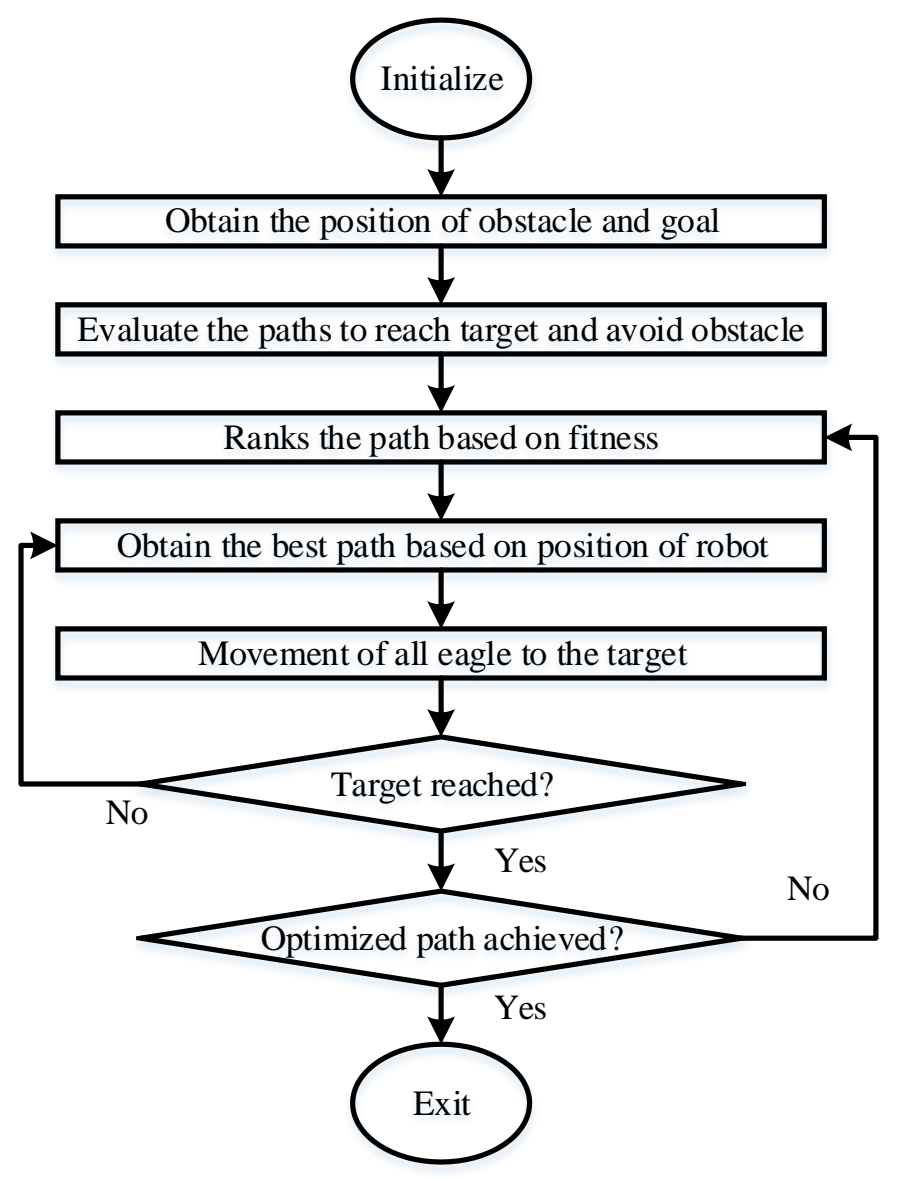

Fig. 1. Flowchart of Eagle strategy for route mapping of humanoid robot 


\subsection{Ant Colony Optimization}

The flowchart of ACO is portrayed in Fig. 2. In the actual field, it is understood that biological ants may use swarm knowledge to explore the shortest path to the food source. Ant Colony Optimization (ACO) techniques are designed to imitate actual ate actions in order to include heuristic optimization strategies. Dorigo [23] has suggested it first in 1992. Biological ants display complicated social behaviors dependent on the hormones accumulated while looking for nutrition (called pheromones). Pheromones encourage other ants and describe the route, which other ants will take to the source of food. More aunts go through the route, and more pheromones are put, and more ants are likely to go through the route. The shortest route to food creates the most pheromones since it can be traveled in less time by more ants. This phenomenon has been first noted in the popular Double Bridge experiment: the ants always prefer the quickest route after a certain time offered the option between a short route and a long route to a food supply. The pheromone can also vaporize through a period to avoid the establishment of a sub-optimal direction (when the solution is at a lower limit), thereby decreasing the transition to other ants. On the other hand, the pheromone concentrations are still large on the shortest course since the deposit rate of pheromone is higher than their evaporation rate in the present scenario.

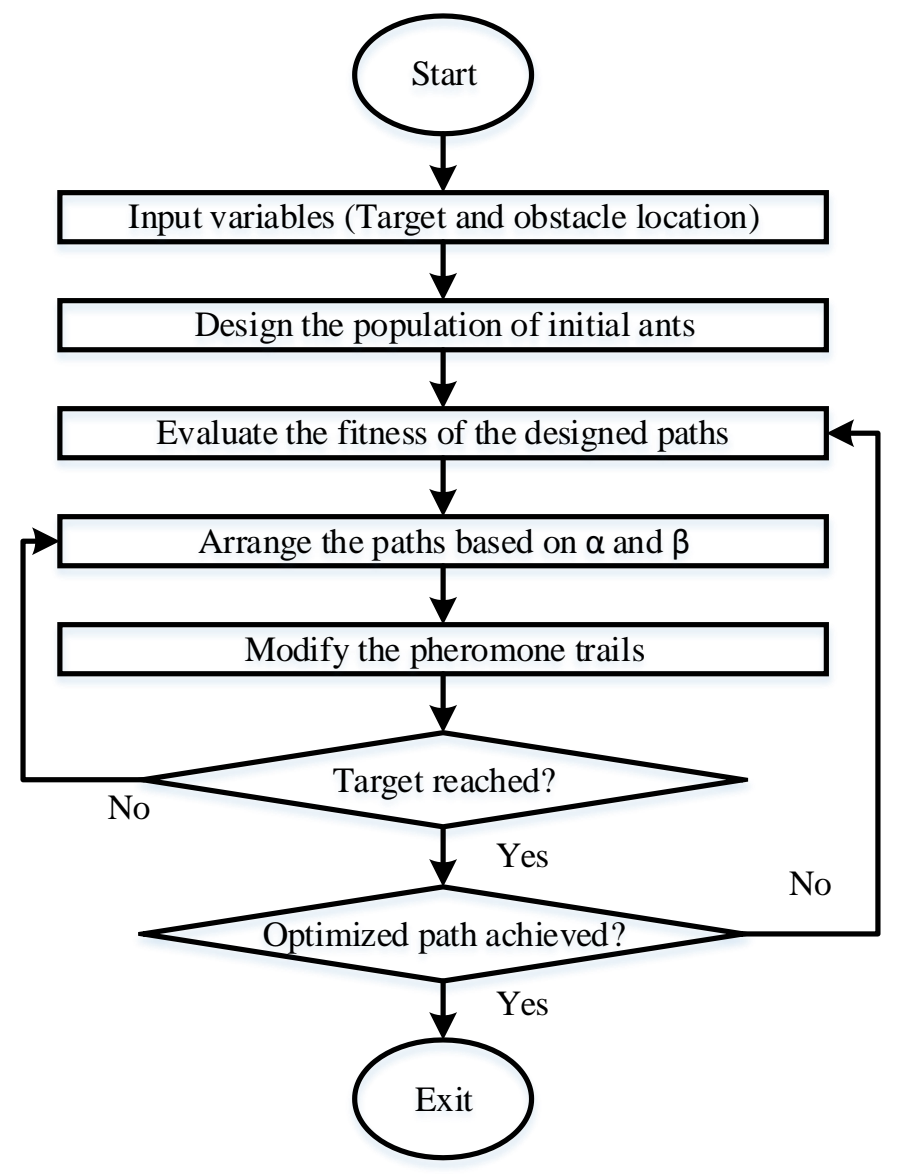

Fig. 2. Flowchart of ant colony optimization for navigation of a humanoid robot

Take into account a network in which ants can migrate between locations. The possibility of an ant $a$ in the node $j$ decides to proceed to another node $k$ with the use of pheromone concentrations $p_{j k}^{a}$ is presented using by [24] as 


$$
\mathrm{P}_{j k}^{a}= \begin{cases}\frac{\left(1_{j k}^{a}\right)^{\alpha}\left(\eta_{j k}^{a}\right)^{\beta}}{\sum_{z \in m_{j k}^{a}}\left(1_{j k}^{a}\right)^{\alpha}\left(\eta_{j k}^{a}\right)^{\beta}} & \text { if } k \in m_{j}^{a} \\ 0 & \text { if } k \notin m_{j}^{a}\end{cases}
$$

The more pheromone in a track, the greater the chance the ant is going to follow the route. In summarizing the denominator, all options (or adjacent modules) in a set $m_{j}^{a}$ are considered when the ant is in the domain $j$. Both $\alpha, \beta$ and $\eta_{j k}^{a}$ are typically based on implementation, where $\eta_{j k}^{a}$ is a heuristic indication, and the $\alpha$ and $\beta$ values evaluate the significance of both heuristic and pheromone factors. When $\beta=0$ then the possibility affects only on pheromone amounts, while when $\alpha=0$ the possibility varies only on heuristic properties, that is, the node nearest to the present node is the most likely to be chosen.

The route's pheromone concentrations (from node I to node ii) will evaporate at a speed of E (also known as the evaporation speed)

$$
1_{j k} \leftarrow(1-\mathrm{E}) 1_{j k}
$$

where $0 \leq \mathrm{E}<1$. After pheromone evaporation, the fresh pheromone concentrations are modified with the incremental pheromone laid down by the ants), which just passed the route with the cost $C^{a}$ of ant $a$ :

$$
\begin{gathered}
1_{j k} \leftarrow 1_{j k}+\sum_{a=1}^{n} \Delta \mathrm{l}_{j k}^{a} \\
\Delta \mathbf{l}_{j k}^{a}=\frac{1}{C^{a}}
\end{gathered}
$$

\subsection{Hybridization of Eagle strategy and Ant colony optimization}

Humanoid navigation is a difficult field to study because it necessitates precision and consistency in a limited amount of time and space. As a result, combining two strategies is often fascinating. To overcome the drawbacks of standalone approaches, ES is combined with ACO in this study. First, the ES receives sensory data about obstacle ranges. The ES generates an interim steering angle dependent on the learning model data given by the user. The ultimate steering is then produced by ACO. The hybridization system used in the present study is shown in Fig. 3. The steps describing the hybridization and providing optimum steering angle are:

1. The inbuilt sensors of the humanoid robot obtain the location of the obstacles and target.

2. It follows a straight-line path to reach the target.

3. As soon the robot detects an obstacle inside its threshold range, the ES gets activated and gives an intermediate steering angle.

4. This angle is then provided to ACO, which gives another steering angle.

5. If the steering angle obtained in step 4 is optimum, the robot will take a turn-based on it. Otherwise, this angle is transferred to step 3.

6. The process will continue till the robot obtain an optimum steering angle to avoid obstacle and reach the target. 


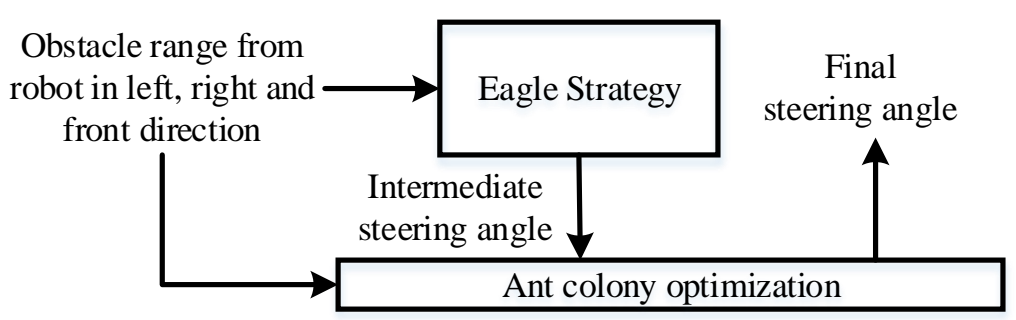

Fig. 3. Mechanism of hybridization of Eagle strategy and Ant colony optimization

\section{Implementation of Proposed Controller in Humanoid NAO}

The evolved hybrid ES-ACO method is used to simulate and test the navigation of a single humanoid NAO in challenging situations. For the simulation process, a 240x160 unit navigational workspace with complicated static obstacles is created in the Webots simulator. Fig. 4 depicts the effects of a single humanoid robot simulation. Fig. 5 depicts the experimental results of single humanoid route planning that is established under controlled circumstances identical to the simulation model for the operation of established research. Table 1 compares and presents the simulation and experimental findings in consideration of process length and process duration. Based on the result obtained through the pictorial and tabular representation, it is clear that the robot is reaching the target using the proposed controller. The deviation obtained is under $5 \%$. It demonstrates that the simulation and experiments are in good agreement, and the simulation results are validated using experimental results.
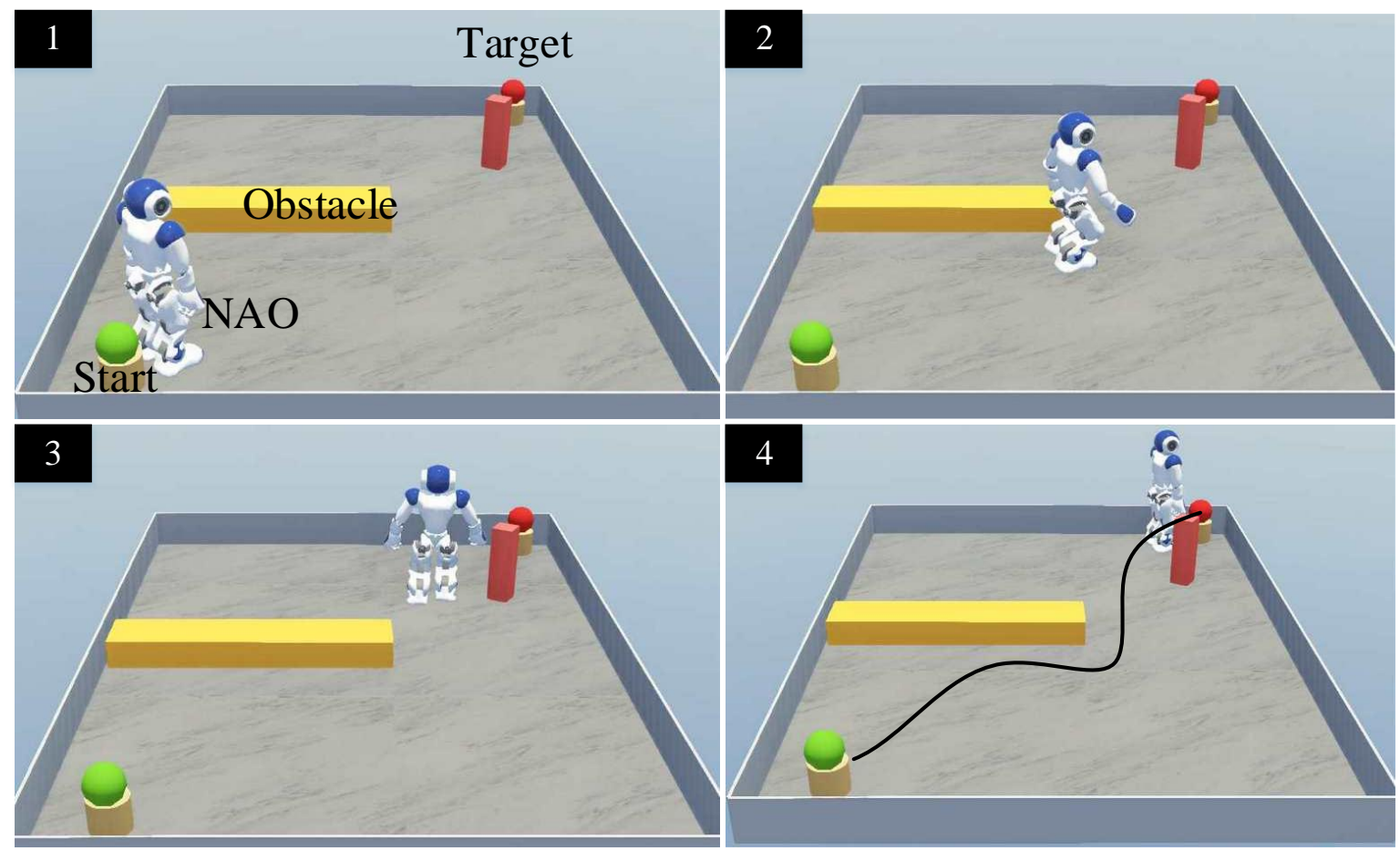

Fig. 4. Snapshots of navigation of humanoid robot in complex simulated terrain 
Table 1. Analysis of deviation between simulation and experimental results of humanoid robot navigation

\begin{tabular}{ccccccc}
\hline \multirow{2}{*}{ Sl. No. } & \multicolumn{2}{c}{ Simulation } & \multicolumn{2}{c}{ Experiment } & \multicolumn{2}{c}{ Deviation (\%) } \\
\cline { 2 - 7 } 1. & 264.7 & 70.95 & 276.5 & 73.57 & 4.46 & 3.69 \\
\hline 2. & 283.7 & 75.96 & 293.8 & 78.82 & 3.56 & 3.77 \\
\hline 3. & 285.8 & 77.42 & 299.9 & 81.18 & 4.93 & 4.86 \\
\hline 4. & 270.5 & 72.95 & 283.9 & 76.16 & 4.95 & 4.4 \\
\hline 5. & 287.7 & 78.17 & 296.3 & 81.85 & 2.99 & 4.71 \\
\hline 6. & 300.9 & 81.18 & 312.2 & 84.91 & 3.76 & 4.59 \\
\hline 7. & 275 & 74.66 & 286.2 & 78.2 & 4.07 & 4.74 \\
\hline 8. & 292.2 & 79.88 & 304.6 & 83.69 & 4.24 & 4.77 \\
\hline 9. & 281.9 & 76.17 & 294.9 & 79.76 & 4.61 & 4.71 \\
\hline 10. & 268.6 & 72.2 & 280.5 & 75.59 & 4.43 & 4.7 \\
\hline Avg. & 281.1 & 75.96 & 292.88 & 79.38 & 4.2 & 4.5 \\
\hline
\end{tabular}

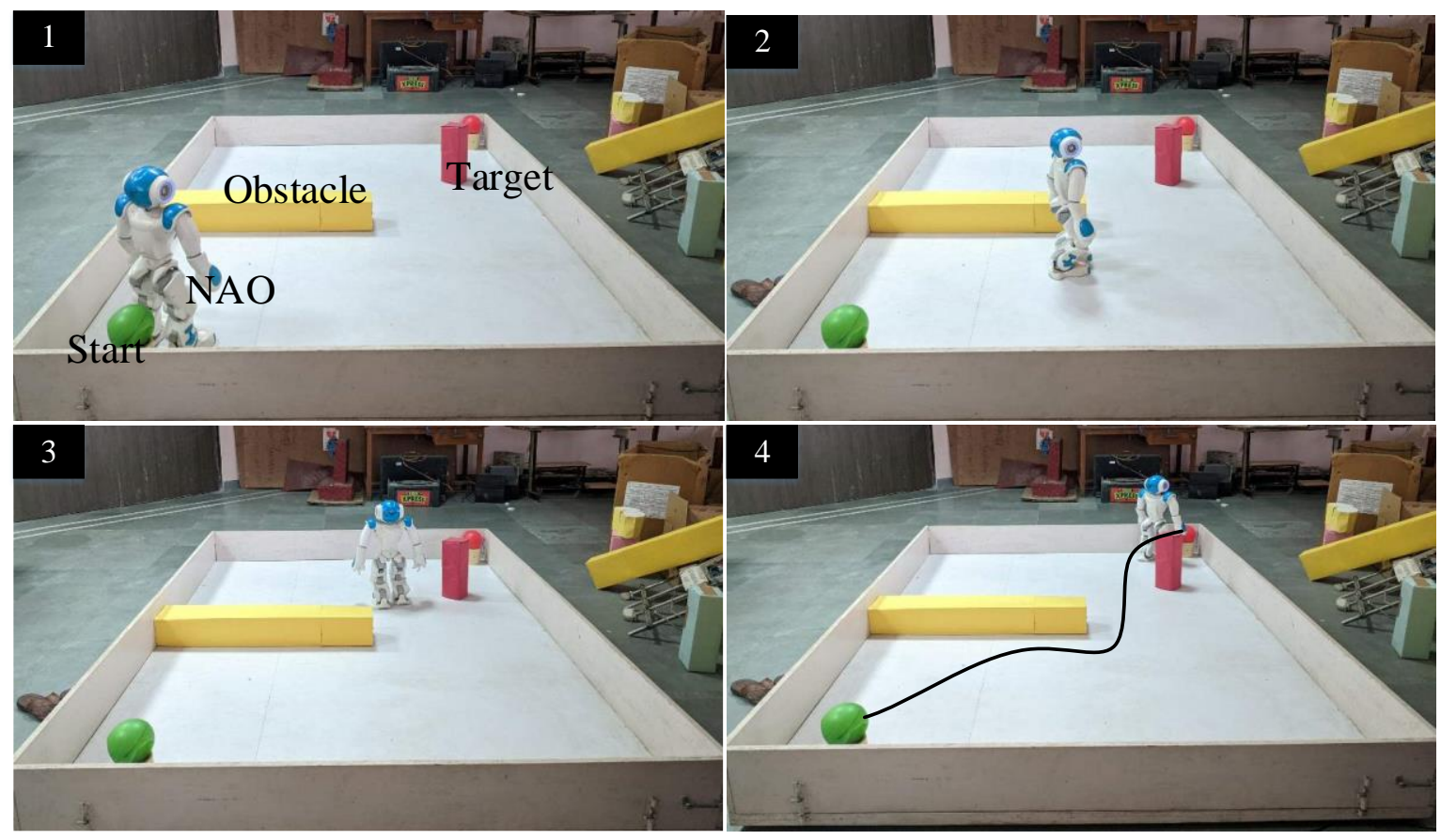

Fig. 5. Snapshots of navigation of humanoid robot in real complex terrain

\section{Conclusion}

The recent research develops and implements a hybrid ES-ACO method for seamless route mapping of single humanoids in complicated terrains. The front, left, and right obstacle ranges from the NAO are transmitted into the hybrid ES-ACO approach, and the precise steering angle is gathered as the outcome. The established control system simulation scenarios are established in Webots to evaluate their efficacy. The findings are evaluated in experimental environments created under controlled circumstances. The findings in both conditions display a high degree of consistency with suitable percentage deviations. This analysis can be widely applied as an artificial intelligence strategy for engineering problem optimization. 


\section{References}

[1] A. K. Kashyap, D. R. Parhi, and S. Kumar, "Dynamic Stabilization of NAO Humanoid Robot Based on Whole-Body Control with Simulated Annealing," Int. J. Humanoid Robot., vol. 17, no. 03, p. 2050014, 2020. https: //doi.org/10.1142/S0219843620500140

[2] A. K. Kashyap and A. Pandey, "Different Nature-Inspired Techniques Applied for Motion Planning of Wheeled Robot: A Critical Review," Int. J. Adv. Robot. Autom., vol. 3, no. 2, pp. 1-10, 2018. https://doi.org/10.15226/2473-3032/3/2/00136

[3] K. P. Lagaza, A. K. Kashyap, and A. Pandey, "Spider Monkey Optimization Algorithm Based CollisionFree Navigation and Path Optimization for a Mobile Robot in the Static Environment," in Advances in Mechanical Engineering, 2020, pp. 1459-1473. https://doi.org/10.1007/978-981-15-0124$1 \_128$

[4] A. K. Kashyap, A. Pandey, A. Chhotray, and D. R. Parhi, "Controlled Gait Planning of Humanoid Robot NAO Based on 3D-LIPM Model," SSRN Electron. J., 2020. https://doi.org/10.2139/ssrn.3552498

[5] A. K. Kashyap and A. Pandey, "Optimized Path Planning for Three-Wheeled Autonomous Robot Using Teaching-Learning-Based Optimization Technique," in Advances in Materials and Manufacturing Engineering, 2020, pp. 49-57. https://doi.org/10.1007/978-981-15-1307-7_5

[6] A. K. Kashyap, K. Pirewa Lagaza, and A. Pandey, "Dynamic Path Planning for Autonomous Mobile Robot using Minimum Fuzzy Rule Based Controller with Avoidance of Moving Obstacles," in 2018 International Conference on Recent Innovations in Electrical, Electronics \& Communication Engineering (ICRIEECE), 2018, p. 3330-3335. https://doi.org/10.1109/ICRIEECE44171.2018.9009120

[7] D. Murray and C. Jennings, "Stereo vision based mapping and navigation for mobile robots," Proceedings of International Conference on Robotics and Automation, Albuquerque, NM, USA, 1997, vol. 2, 1997, pp. 1694-1699. https://doi.org/10.1109/ROBOT.1997.614387

[8] B.B.V.L. Deepak, D.R. Parhi, S. Kundu, "Innate Immune based Path Planner of an Autonomous Mobile Robot," Procedia Eng., vol. 38, 2012, pp. 2663-2671. https://doi.org/10.1016/j.proeng.2012.06.313

[9] J.-A. Meyer and D. Filliat, "Map-based navigation in mobile robots," Cogn. Syst. Res., vol. 4, no. 4, pp. 283-317, 2003. https://doi.org/10.1016/S1389-0417(03)00007-X

[10] K. H. Kowdiki, R. K. Barai, and S. Bhattacharya, "Leader-follower formation control using artificial potential functions: A kinematic approach," IEEE-International Conf. Adv. Eng. Sci. Manag. ICAESM2012, pp. 500-505, 2012. https://ieeexplore.ieee.org/abstract/document/6216054

[11] P. Shi and Y. Zhao, "An efficient path planning algorithm for mobile robot using improved potential field," in 2009 IEEE International Conference on Robotics and Biomimetics (ROBIO), 2009, pp. 17041708. https: //doi.org/10.1109/ROBIO.2009.5420407

[12] J. Sfeir, M. Saad, and H. Saliah-Hassane, "An improved Artificial Potential Field approach to realtime mobile robot path planning in an unknown environment," ROSE 2011 - IEEE Int. Symp. Robot. Sensors Environ. Proc., pp. 208-213, 2011. https://doi.org/10.1109/ROSE.2011.6058518

[13] H. Choset and P. Pignon, "Coverage Path Planning: The Boustrophedon Cellular Decomposition," F. Serv. Robot., pp. 203-209, 1998. https://doi.org/10.1007/978-1-4471-1273-0_32

[14] L. E. Kavraki, P. Švestka, J. C. Latombe, and M. H. Overmars, "Probabilistic roadmaps for path planning in high-dimensional configuration spaces," IEEE Trans. Robot. Autom., vol. 12, no. 4, pp. 566-580, 1996. https://doi.org/10.1109/70.508439

[15] T. A. Cargill, "A robust distributed solution to the dining philosophers problem," Softw. Pract. Exp., vol. 12, no. 10, pp. 965-969, 1982. https://doi.org/10.1002/spe.4380121009

[16] H. Wedde, "A starvation-free solution of the dining philosophers' problem by use of interaction systems," in International Symposium on Mathematical Foundations of Computer Science, 1981, pp. 534-543. https://doi.org/10.1007/3-540-10856-4_122 
[17] A. Pandey, A. K. Kashyap, D. R. Parhi, and B. K. Patle, "Autonomous mobile robot navigation between static and dynamic obstacles using multiple ANFIS architecture," World J. Eng., vol. 16, no. 2, pp. 275-286, 2019. https://doi.org/10.1108/WJE-03-2018-0092

[18] A. K. Kashyap, D. R. Parhi, M. K. Muni, and K. K. Pandey, "A hybrid technique for path planning of humanoid robot NAO in static and dynamic terrains," Appl. Soft Comput., vol. 96, p. 106581, 2020. https://doi.org/10.1016/j.asoc.2020.106581

[19] A. K. Kashyap, D. Parhi, and A. Pandey, "Improved Modified Chaotic Invasive Weed Optimization Approach to Solve Multi-Target Assignment for Humanoid Robot," J. Robot. Control, vol. 2, no. 3, pp. 194-199, 2021. https://doi.org/10.18196/jrc.2377

[20] A. K. Kashyap and D. R. Parhi, "Dynamic walking of humanoid robot on flat surface using amplified LIPM plus flywheel model," International Journal of Intelligent Unmanned Systems, vol. ahead-of-p, no. ahead-of-print. 2021. https://doi.org/10.1108/IJIUS-09-2020-0039

[21] A. K. Kashyap and D. R. Parhi, "Particle Swarm Optimization aided PID gait controller design for a humanoid robot," ISA Trans., 2020. https://doi.org/10.1016/j.isatra.2020.12.033

[22] H. Yapıcı and N. Çetinkaya, "An Improved Particle Swarm Optimization Algorithm Using Eagle Strategy for Power Loss Minimization," Math. Probl. Eng., vol. 2017, pp. 1-11, 2017. https://doi.org/10.1155/2017/1063045

[23] M. Dorigo, V. Maniezzo, and A. Colorni, "Ant system: Optimization by a colony of cooperating agents," IEEE Trans. Syst. Man, Cybern. Part B Cybern., vol. 26, no. 1, pp. 29-41, 1996. https://doi.org/10.1109/3477.484436

[24] A. K. Kashyap and D. R. Parhi, "Optimization of stability of humanoid robot NAO using ant colony optimization tuned MPC controller for uneven path," Soft Comput., vol. 1, 2021. https://doi.org/10.1007/s00500-020-05515-1 